\title{
Phorbol Myristate Acetate, Dioctanoylglycerol, and Phosphatidic Acid Inhibit the Hydroosmotic Effect of Vasopressin on Rabbit Cortical Collecting Tubule
}

\author{
Yasuhiro Ando, Harry R. Jacobson, and Matthew D. Breyer \\ Division of Nephrology, Department of Medicine, Vanderbilt University, Nashville, Tennessee 37232
}

\begin{abstract}
We explored the role for protein kinase $\mathrm{C}$ (PKC) in modulating vasopressin (AVP)-stimulated hydraulic conductivity (Lp) in rabbit cortical collecting tubule (CCT) perfused in vitro at $37^{\circ} \mathrm{C}$. In control studies, $10 \mu \mathrm{U} / \mathrm{ml}$ AVP increased Lp (mean \pm SE, $\times 10^{-7}$ centimeters/atmosphere per second) from $4.4 \pm 0.9$ to 166.0 \pm 10.4 . Pretreatment with dioctanoylglycerol (DiC8) suppressed AVP stimulated peak Lp (peak Lp, 21.9 \pm 3.1). Pretreatment with $10^{-9}$ and $10^{-7} \mathrm{M} 4 \beta$-phorbol $12 \beta$-myristate $13 \alpha$ acetate (PMA) also blocked the increase in $L_{p}$ in a dose-dependent fashion (peak $L p, 59.3 \pm 7.5$ and $18.6 \pm 4.8$, respectively). Inactive phorbol ester, $4 \alpha$-phorbol $12 \beta, 13 \alpha$-didecanoate $\left(10^{-7}\right.$ $M)$, had no effect. PMA also suppressed the increase in $L_{p}$ induced by $10^{-4} \mathrm{M}$ 8-p-chlorophenylthio-cyclic AMP (CcAMP): peak $L p$ was $169.4 \pm 14.9$ in control, $79.2 \pm 5.5$ with $10^{-9} \mathrm{M}$ PMA, and 25.7 \pm 2.9 with $10^{-7} \mathrm{M}$ PMA. Furthermore, when $10^{-7} \mathrm{M}$ PMA was added to the bath $10 \mathrm{~min}$ after exposure to AVP, the Lp response to AVP was blocked. Peak Lp was 52.4 99.6 with PMA vs. $165.1 \pm 10.0$ in control.

Phosphatidic acid (PA), which is thought to stimulate phosphatidylinositol (PI) turnover, produced similar inhibitory effects on AVP as well as CcAMP-stimulated Lp: PA suppressed 10$\mu \mathrm{U} / \mathrm{ml} \mathrm{AVP}$-induced peak $\mathrm{Lp}$ from a control value of $159.6 \pm 7.9$ to $88.9 \pm 15.8$, and $10^{-4} \mathrm{M}$ CcAMP induced peak $\mathrm{Lp}$ from $169.4 \pm 14.9$ to $95.5 \pm 7.7$.

We conclude that (a) PMA, at concentrations known to specifically activate PKC, suppresses the hydroosmotic effect of AVP on CCT; (b) This suppression is primarily a post-cAMP event; (c) Inhibition of AVP-stimulated Lp by DiC8 and PA also suggests an inhibitory role for the PKC system; $(d)$ The ability of pre- and post-AVP administration of PMA to blunt the AVP response suggests that agents that act through modulation of PI turnover in CCT may regulate the hydroosmotic effect of AVP.
\end{abstract}

Parts of this study were presented at the annual meeting of the Federation of American Societies for Experimental Biology, 1987, in Washington, D. C., and at the American Federation of Clinical Research, 1987, in San Diego.

Address reprint requests to Dr. Breyer, Div. of Nephrology, Vanderbilt University, Nashville, TN 37232.

Received for publication 6 February 1987 and in revised form 13 April 1987.

J. Clin. Invest.

(c) The American Society for Clinical Investigation, Inc. 0021-9738/87/08/0590/04 \$2.00

Volume 80, August 1987, 590-593
Introduction

Calcium-activated, phospholipid-dependent protein kinase $\mathrm{C}$ (PKC) $)^{1}$ is widely distributed in mammalian tissues $(1,2)$, and is considered an integral component of an intracellular signal transduction pathway used by numerous hormones and biologically active substances $(3,4)$. Interactions between the PKC pathway and the better-characterized pathway mediated by cyclic adenosine monophosphate (cAMP) is a topic of great physiological importance. To test for this interaction in a system relevant to the kidney, we examined the effect of dioctanoylglycerol (DiC8), phorbol esters, and phosphatidic acid (PA) on the hydroosmotic response of the rabbit cortical collecting tubule (CCT) to arginine vasopressin (AVP), which is a classic cAMP-mediated response.

Our findings suggest that activation of PKC antagonizes the effect of AVP on the rabbit CCT predominantly via a post-cAMP action.

\section{Methods}

Female New Zealand white rabbits weighing $1.5-2.0 \mathrm{~kg}$ were killed by decapitation. The left kidney was removed and 1-mm-thick coronal slices were made. These slices were placed in ice-chilled medium that had the same composition as dissection medium described below. A slice in dissection medium was transferred to an ice-chilled stage of a dissection microscope and individual CCTs were dissected with sharpened forceps. The isolated CCT (1.0-2.5 mm in length) was then transferred to a thermostatically controlled lucite bath chamber on an inverted microscope stage. The tubule was cannulated and perfused with concentric micropipettes as previously described (5-7). Generally CCTs were mounted in the perfusion set up within $15 \mathrm{~min}$ after exsanguinating the animal.

After cannulation of a tubule, flow rate of bath solution was adjusted to $0.5 \mathrm{ml} / \mathrm{min}$ with an infusion pump (Sage Instruments, Inc., Cambridge, MA). Transepithelial potential difference was monitored with an electrometer (model 602; Keithley Instruments, Inc., Cleveland, $\mathrm{OH}$ ) and recorded on a strip chart recorder (Primeline model R-02; Soltec Co., Sun Valley, CA).

The bath medium was warmed to $37^{\circ} \mathrm{C}$. To retain an effective transepithelial osmotic gradient along the entire length of the tubule, the perfusion rate was adjusted to $15-20 \mathrm{nl} / \mathrm{min}$. The perfused fluid was

1. Abbreviations used in this paper: AA, arachidonic acid; AVP, arginine vasopressin; $\left[\mathrm{Ca}^{++}\right] \mathrm{i}$, intracellular calcium ion concentration; CcAMP, 8-(p-chlorophenylthio)-cyclic AMP; CCT, cortical collecting tubule; DiC8, dioctanoylglycerol; Jv, net volume flux; Lp, hydraulic conductivity; PA, phosphatidic acid; PDD, $4 \alpha$-phorbol 12 $\beta, 13 \alpha$-didecanoate; PG, prostaglandin; $\mathrm{PH}$, intracellular $\mathrm{pH}$; PKC, protein kinase C; PMA, $4 \beta$ phorbol $12 \beta$-myristate $13 \alpha$-acetate. 
collected in a constriction pipette of known volume (between 80 and $150 \mathrm{nl})$.

Composition of bath medium, dissection medium, and isotonic perfusate was as follows (in millimolars): $\mathrm{NaCl}, 105, \mathrm{NaHCO}_{3}, 25, \mathrm{Na}$ acetate, $10, \mathrm{NaHPO}_{4}, 2.3, \mathrm{KCl}, 5, \mathrm{CaCl}_{2}, 1.8, \mathrm{MgSO}_{4}, 1.0$, glucose, 8.3, and alanine, 5 (osmolality 300 mosmol). Before use, all solutions were bubbled to equilibration at $37^{\circ} \mathrm{C}$ with $95 \% \mathrm{O}_{2} / 5 \% \mathrm{CO}_{2}$ gas mixture to achieve a $\mathrm{pH}$ of 7.40 . Bath medium was put in a silicon-coated glass syringe mounted on the infusion pump. Composition of hypotonic perfusate was the same except for the low osmolality (150 mosmol) due to lower $\mathrm{NaCl}$ concentration $(30 \mathrm{mM})$ and the addition of tritium-labeled inulin as a volume marker. Both isotonic and hypotonic perfusate contained $0.2 \mathrm{mg} / \mathrm{ml}$ Food, Drug and Cosmetic dye No. 3 (Aniline and Chemical Co., Chicago, IL) to detect cell damage and perfusate leak (8).

Net volume flux (Jv, nanoliters per minute per millimeter) was calculated for each collection as follows:

$$
\begin{aligned}
V o & =V p / t, \\
V i & =V o \times C o / C i, \text { and } \\
J v & =(V i-V o) / L,
\end{aligned}
$$

where $V o$ and $V i$ are collection and perfusion rate (nanoliters per minute), respectively, $V p$ is volume of constriction pipette (nanoliters), $t$ is collection time (minute), $\mathrm{Co}$ and $\mathrm{Ci}$ are radioactivity of tritium-labeled inulin (counts per minute per nanoliter) in collected fluid and perfusate, respectively, and $L$ is tubule length (millimeters). $C o$ and $C i$ were counted by liquid scintillation counter (model 6892; Tracor Analytic Co., Des Plaines, IL). $L$ is measured directly by eyepiece reticle at the end of each experiment.

Hydraulic conductivity (Lp, centimeters per atmosphere [atm] per second) was calculated according to Du Bois et al. (9):

$(1 / R T S) \cdot(1 / O b)^{2} \cdot[O b \cdot(V i-V o)+O i \cdot V i$

$\times \operatorname{Ln}\{(\mathrm{Ob}-\mathrm{Oi}) \cdot \mathrm{Vi} /(\mathrm{Ob} \cdot \mathrm{Vo}-\mathrm{O} i \cdot \mathrm{Vi})\}]$,

where $R$ is the gas constant, $T$ is temperature of bath medium (degree kelvin), $S$ is luminal surface area of the tubule calculated using an assumed lumen diameter of $20 \mu \mathrm{m}$, and $O b$ and $O i$ are osmolality of bath medium and perfusate, respectively.

\section{Experimental protocols}

During the first 30-45 min of incubation in all experiments, tubules were perfused with isotonic perfusate. Subsequently perfusate was changed to hypotonic perfusate.

1. Effect of DiC8 and phorbol esters on AVP response. After the incubation for $90 \mathrm{~min}$, three collections were made for calculation of basal Jv and Lp. Then $10 \mu \mathrm{U} / \mathrm{ml}$ AVP was added to the bath and six to seven collections were made during the 15-50 min after exposure to AVP to determine peak Jv and Lp. This submaximal AVP dose was chosen to theoretically enable us to see either suppressive or stimulatory effects of the agents examined on AVP response. Peak Jv and Lp were defined as mean of the three largest values during exposure to AVP. Peak was usually observed $25-45 \mathrm{~min}$ after AVP exposure.

In experimental studies, DiC8 (50 $\mu \mathrm{g} / \mathrm{ml}), 10^{-7}-10^{-11} \mathrm{M} 4 \beta$-phorbol $12 \beta$-myristate $13 \alpha$-acetate (PMA) or $10^{-7} \mathrm{M} 4 \alpha$-phorbol $12 \beta, 13 \alpha$-didecanoate (PDD) was added to bath medium 10-15 min before the basal collections. To avoid degeneration of phorbol esters, the syringe mounted on the pump was wrapped with aluminum foil. AVP was then added and peak $\mathrm{Jv}$ and Lp determined as above. Pure dimethylsulphoxide (DMSO) was used as solvent for the phorbol esters and the final concentration of DMSO in bath medium of all experiments was $0.01 \%$ by volume. $\mathrm{DiC} 8$ was also dissolved in DMSO and dispersed by sonication after being diluted into the medium. In DMSO control studies, identical amounts of DMSO without phorbol esters or DiC8 was added to bath medium.
2. Effect of PMA on post-cAMP events. $10^{-4} \mathrm{M} 8$-( $p$-chlorophenylthio)cyclic AMP (CcAMP) was added instead of AVP with or without $10^{-7}$ or $10^{-9} \mathrm{M}$ PMA pretreatment. DMSO control studies were pretreated with $0.01 \%$ DMSO in the same manner described above.

3. Effect of PMA posttreatment on AVP response. In these studies, $0.01 \%$ DMSO was added to the bath $15 \mathrm{~min}$ before the basal collections. Then, $10 \mathrm{~min}$ after the exposure to $10 \mu \mathrm{U} / \mathrm{ml} \mathrm{AVP,} 10^{-7} \mathrm{M}$ PMA was added to bath medium. Collections were started soon after the addition of PMA and six or seven collections were made during the 10-50 min after the AVP exposure. Peak Jv and Lp of both control and experimental studies were calculated as mean of the three largest values during the 30-50 min after AVP exposure. The DMSO control studies in protocol 1 served as the control group.

4. Effects of $P A$ on $A V P$ and CCAMP response. $\mathrm{L}-\alpha-\mathrm{PA}$ (99\% pure, $\mathrm{Na}$ salt) was used in place of PMA. The control studies in protocol 1 and the DMSO control studies in protocol 2 served as the control for the AVP and CcAMP studies, respectively. In experimental studies, 25 $\mu \mathrm{g} / \mathrm{ml}$ PA was added to bath medium instead of PMA. An aqueous dispersion of $5 \mathrm{mg} / \mathrm{ml} \mathrm{PA}$ was briefly sonicated and added to bathing medium just before the experiment to give the final concentration indicated above.

\section{Reagents}

AVP, DiC8, PMA, PDD, DMSO, CcAMP, and PA were purchased from Sigma Chemical Co., St. Louis, MO. Radioactive tritium-labeled inulin was purchased from DuPont NEN Products Co., Boston, MA.

\section{Statistics}

Data are presented as mean $\pm \mathrm{SE}$, and statistical comparisons were made using non-paired $t$ test. Difference with $P<0.05$ was considered statistically significant.

\section{Results}

As Jv and Lp are qualitatively the same as a parameter of water transport, only Lp's are presented below.

1. Effect of DiC8 and phorbol esters on AVP response (Figs. 1 and 2). DiC8 $(50 \mu \mathrm{g} / \mathrm{ml})$ slightly increased basal $\mathrm{Lp}(P<0.02)$ and suppressed peak $\operatorname{Lp}(P<0.001)$. In addition, $10^{-9}$ and $10^{-7}$ M PMA suppressed peak Lp in a dose-dependent fashion $(P$ $<0.01$ and $P<0.001$, respectively). At $10^{-7} \mathrm{M}$, PMA completely suppressed the AVP response. $10^{-11}$ and $10^{-10} \mathrm{M}$ PMA had no significant effect on basal or peak Lp. Basal Lp was increased slightly by $10^{-7}$ M PMA, but because of the large standard deviation, this increase was not statistically significant. Inactive phorbol ester, PDD, had no effect on either basal or peak Lp even at $10^{-7} \mathrm{M}$. The solvent, DMSO, at $0.01 \%$ by volume had no effect on basal or peak $\mathrm{Lp}$.

2. Effect of PMA on CcAMP response (Fig. 2). $10^{-9}$ and $10^{-7}$ $M$ PMA suppressed peak $L p$ induced by CcAMP in a dose-

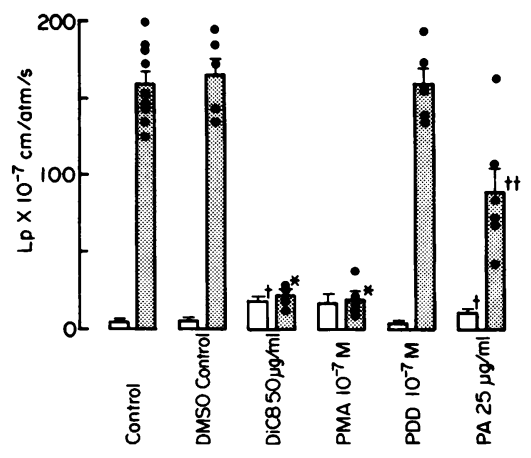

Figure 1. Effect of DMSO, DiC8, phorbol esters, and PA on basal and peak Lp. Data are presented as mean $\pm \mathrm{SE}$ (Open bar) Basal Lp. (Shaded bar) Peak Lp induced by $10 \mu \mathrm{U} / \mathrm{ml}$ AVP. Each dot represents an individual experiment. $\dagger, P<.02 ; \mathrm{t}$, $P<.01 ; *, P<.001$ vs. corresponding control. 


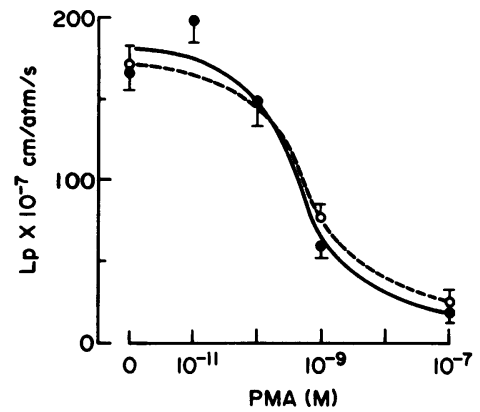

Figure 2. Dose dependence of the PMA effect on peak Lp induced by $10 \mu \mathrm{U} / \mathrm{ml}$ AVP (closed circles) and $10^{-4} \mathrm{M}$ CcAMP (open circles). Each data point represents the mean $\pm S E$ of five experiments.

dependent fashion (both $P<0.001$ ). $10^{-7} \mathrm{M}$ PMA also induced a small but significant $(P<0.05)$ increase in basal Lp; basal Lp $\left(\times 10^{-7} \mathrm{M} \mathrm{cm} /\right.$ atm per s) was $8.5 \pm 1.0(n=5)$ with PMA and 4.3 $\pm 1.1(n=5)$ in DMSO control.

3. Effect of PMA posttreatment on AVP response. $10^{-7} \mathrm{M}$ PMA administrated 10 min after exposure to AVP significantly suppressed the subsequent increase in Lp induced by AVP ( $P$ $<0.001)$; peak $\mathrm{Lp}\left(\times 10^{-7} \mathrm{~cm} /\right.$ atm per s) was $52.4 \pm 9.6(n=6)$ with PMA and $165.1 \pm 10.0(n=5)$ in DMSO control.

4. Effect of PA on AVP and CcAMP response. PA $(25 \mu \mathrm{g} /$ ml) suppressed AVP-induced peak Lp. Basal Lp was increased to a small but significant degree (Fig. 1) $(P<0.02)$. PA also blocked the Lp response to $10^{-4} \mathrm{M}$ CcAMP $(P<0.01)$; peak Lp $\left(\times 10^{-7} \mathrm{~cm} /\right.$ atm per s) was $95.5 \pm 7.7(n=5)$ while control peak Lp was $169.4 \pm 14.9(n=5)$.

\section{Discussion}

Numerous hormones and biologically active substances have been shown to stimulate PI turnover and activate PKC (10). The role for PKC in modulating cell functions has been intensely investigated in a variety of cell systems $(1-4,10,11)$. The interactions between the PKC pathway and other signal transduction systems, especially the cAMP (or protein kinase A) pathway, are not well characterized. In thrombocytes, cAMP inhibits PKC-mediated serotonin release (12). In hepatic cells, cAMP and PKC independently stimulate glycogenolysis (3). Potential modulation by PKC of the CAMP pathway has not been well documented.

The hydroosmotic effect of AVP on mammalian CCT (13) or amphibian urinary bladder (14) represents a typical cAMPmediated system. The present studies have examined the potential interaction between PKC and response of the CCT to AVP using PMA, a highly specific activator of PKC (15). Our result clearly revealed a powerful inhibitory effect of PMA on the hydroosmotic effect of AVP (Figs. 1 and 2). Since microassay for PKC activity has not as yet been developed, we were unable to directly measure the PKC activity in CCT. However, PMA exerted a suppressive effect in a dose-dependent manner, using nanomolar concentrations, which were previously shown to specifically activate $\operatorname{PKC}(3,11)$. Furthermore, identical concentration of PDD, which is structurally similar to PMA but ineffective in activating PKC (15), had no suppressive effect. We therefore attribute this inhibition to PKC activation.

DiC8 is a synthetic diacylglycerol that has been found to stimulate PKC in a manner similar to endogenous diacylglycerols (16). While chemically dissimilar from PMA, DiC8 also inhibited AVP-stimulated hydroosmotic water flow, arguing for an inhibitory effect of PKC on this pathway.
The suppressive effect of PA (Fig. 1) is also consistent with this inference. PA has recently been found to stimulate PI breakdown by apparently acting as an activator of phospholipase $\mathrm{C}$ $(17,3)$. This results in both inositol triphosphate-mediated increase in intracellular free calcium ion concentration $\left(\left[\mathrm{Ca}^{++}\right] \mathrm{i}\right)$ and diacylglycerol-mediated PKC activation (17). Thus, while direct activation of PKC by PMA is not a naturally occurring event, PA may mimic the physiological stimulation of receptorlinked PI turnover and PKC activation (17).

Recent functional studies in non-mammalian urinary epithelia have demonstrated an inhibitory effect of high dose PMA on AVP-induced water flow $(16,18)$. However, evidence regarding the level at which PKC interferes with AVP action on urinary epithelia are conflicting. Schlondorf and Levine (16) concluded that the site of inhibition by PKC was primarily precAMP. But in their studies, a post-cAMP inhibition was observed in experiments with DiC8. Yorio et al. (18) thought the site of inhibition was post-cAMP. However, they required micromolar concentrations of PMA to demonstrate a post-cyclic AMP inhibition. PKC is maximally stimulated by nanomolar concentration of PMA (3), and higher concentrations as used in previous studies may exert diverse effects in addition to activation of PKC (11). So it is very important to use appropriate concentrations of PMA to exclusively examine the action of PKC. Although we can not exclude the possibility of a co-existant pre-cAMP inhibition as suggested by Dixon et al. in cultured rabbit CCT cells using $10^{-6}$ M PMA (19), our studies with CcAMP (Fig. 2) demonstrate a potent post-cAMP inhibitory effect. To our knowledge, this is the first convincing evidence in epithelia for a post-cAMP suppression by nanomolar PMA. We also found that PMA could block the induction of AVP-stimulated water flux when it was administered after AVP. These results strongly suggest that agents which stimulate PI turnover and PKC in CCT may antagonize AVP action in vivo.

The specific mechanisms by which PKC inhibits AVP- and cAMP-induced increase in water flux in CCT remain undefined. Of the many potential mechanisms, three possible candidates can be examined using the isolated renal tubule: $(i)$ increased synthesis of arachidonic acid (AA) metabolites (i.e., products of cyclooxygenase, lipoxygenase, or epoxygenase pathways), (ii) changes in $\left[\mathrm{Ca}^{++}\right] \mathrm{i}$, and (iii) changes in intracellular $\mathrm{pH}(\mathrm{pHi})$. PMA is known to stimulate AA metabolism $(20,21)$, and prostaglandins (PGs) are known to modulate AVP response in CCT $(22,23)$ and in toad bladder $(24)$. However, PGs are thought to inhibit pre-cAMP steps in rabbit CCT $(22,23)$. Furthermore, the inhibitory effect of PMA on AVP-stimulated water flux is not blocked by the pretreatment with inhibitor of PG synthesis in toad bladder (16). It is possible, however, that PGs (24) or other AA metabolites might act at post-cAMP steps. Calcium is another important modulator of cell function (4). PMA increases $\left[\mathrm{Ca}^{++}\right] \mathrm{i}$ in some cell types $(25,26)$. In addition, increased $\left[\mathrm{Ca}^{++}\right]$i suppresses AVP-induced water flux in frog urinary bladder (27).

Finally, it is clear that pHi modulates several cell functions $(28,29)$. In a variety of cell types, activation of PKC causes increased pHi by stimulating amiloride-sensitive $\mathrm{Na}^{+} / \mathrm{H}^{+}$antiport (28). In toad bladder, changes in pHi modulate AVP-stimulated water flow (30); and, in fibroblasts, blockade of amiloridesensitive $\mathrm{Na}^{+} / \mathrm{H}^{+}$antiport abolishes DNA synthesis stimulated by growth factor, a stimulator of PI turnover (31). Based on the above arguments, the mechanism of PMA-induced inhibition 
of AVP response, although still unclear, could be multifactorial. We are currently studying these possibilities.

In summary, our present study demonstrates that DiC8, nanomolar PMA, and PA suppress AVP action. This effect is primarily a post-cAMP event. Inhibition of the AVP response by pre- and post-treatment with PMA suggests that agents which stimulate PI turnover and activate PKC may modulate the action of AVP in the CCT.

\section{Acknowledgments}

The authors wish to thank Ms. Deborah Asbell-Gillespie for her skillful technical assistance and Ms. Nadine Cline for typing the manuscript.

This study was supported by Veterans Administration grant 151JAI733 and National Institutes of Health grants AM-37097 and DK34056-02.

\section{References}

1. Takai, Y., A. Kishimoto, Y. Isawa, Y. Kawahara, T. Mori, and Y. Nishizuka. 1979. Calcium-dependent activation of a multifunctional protein kinase by membrane phospholipid. J. Biol. Chem. 254:36923695.

2. Minakuchi, R., Y. Takai, B. Yu, and Y. Nishizuka. 1981. Widespread occurrence of calcium-activated, phospholipid-dependent protein kinase in mammalian tissues. J. Biochem. 89:1651-1654.

3. Takai, Y., U. Kikkawa, K. Kaibuchi, and Y. Nishizuka. 1984. Membrane phospholipid metabolism and signal transduction for protein phosphorylation. In Advances in Cyclic Nucleotide and Protein Phosphorylation Research. Vol. 18. P. Greengard and G. A. Robinson, editors. Raven Press, New York, 119-158.

4. Rasmussen, H., I. Kojima, K. Kojima, W. Zawalich, and W. Apfeldort. 1984. Calcium as intracellular messenger. sensitivity modulation, C-kinase pathway, and sustained cellular response. In Advances in Cyclic Nucleotide and Protein Phosphorylation Research. Vol. 18. P. Greengard and G. A. Robinson, editors. Raven Press, New York, 159-193.

5. Burg, M., J. Grantham, M. Abramow, and J. Orloff. 1966. Preparation and study of fragments of single rabbit nephrons. Am. J. Physiol. 210:1293-1298.

6. Jacobson, H. R. 1984. Medullary collecting duct acidification: effects of potassium, $\mathrm{HCO}_{3}^{-}$concentration, and $\mathrm{PCO}_{2}$. J. Clin. Invest. 74:2107-2114.

7. Breyer, M. D., J. P. Kokko, H. R. Jacobson. 1986. Regulation of net bicarbonate transport in rabbit cortical collecting tubule by peritubular $\mathrm{pH}$, carbon dioxide tension, and bicarbonate concentration. J. Clin. Invest. 77:1650-1660.

8. Tabei, K., D. J. Levenson, B. M. Brenner. 1983. Early enhancement of fluid transport in rabbit proximal straight tubules after loss of contralateral renal excretory function. J. Clin. Invest. 72:871-881.

9. DuBois, R., A. Verniory, and M. Abramow. 1976. Computation of the osmotic water permeability of perfused tubule segments. Kidney Int. 10:478-479.

10. Mitchell, R. H. 1983. Polyphosphoinositide breakdown as the initiating reaction in receptor stimulated inositol phospholipid metabolism. Life Sci. 32:2083-2085.

11. Nishizuka, Y. 1984. The role of protein kinase $C$ in cell surface signal transduction and tumor promotion. Nature (Lond.). 308:693-698.

12. Takai, Y., K. Kaibuchi, K. Sano, and Y. Nishizuka. 1982. Counteraction of calcium-activated, phospholipid-dependent protein kinase activation by adenosine $3^{\prime}, 5^{\prime}$-monophosphate and guanosine $3^{\prime}, 5^{\prime}$-monophosphate in platelets. J. Biochem. 91:403-416.
13. Grantham, J. J., and M. B. Burg. 1966. Effect of vasopressin and cyclic AMP on permeability of isolated collecting tubules. Am. J. Physiol. 211:255-259.

14. Orloff, J., and J. S. Handler. 1962. The similarity of effect of vasopressin, adenosine 3',5'-monophosphate (cyclic AMP) and theophylline on the toad bladder. J. Clin. Invest. 41:702-709.

15. Castagna, M., Y. Takai, K. Kaibuchi, K. Sano, U. Kikkawa, and Y. Nishizuka. 1982. Direct activation of calcium-activated, phospholipiddependent protein kinase by tumor-promoting phorbol esters. J. Biol. Chem. 257:7847-7851.

16. Schlondorff, D., and D. Levine. 1985. Inhibition of vasopressin stimulated water flow in toad bladder by phorbol myristate acetate, dioctanoylglycerol, and RHC-80267. J. Clin. Invest. 76:1071-1078.

17. Moolenaar, W. H., W. Kruijer, B. C. Tilly, I. Verlaan, A. J. Bierman, S. W. de Laat. 1986. Growth factor-like action of phosphatidic acid. Nature (Lond.). 323:171-173.

18. Yorio, T., E. Quist, and R. A. Masaracchia. 1985. Calcium phospholipid dependent protein kinase and its relationship to antidiuretic hormone in toad urinary bladder epithelium. Biochem. Biophys. Res. Commun. 133:717-723.

19. Dixon, B. S., R. Breckon, C. Burke, and R. J. Anderson. 1985. Evidence of inhibition of vasopressin stimulated adenylate cyclase activity by activators of protein kinase $\mathrm{C}$ in cultured rabbit cortical collecting tubules. Kidney Int. 29:415. (Abstr.)

20. Ohuchi, K., and L. Levine. 1978. Stimulation of prostaglandin synthesis by tumor-promoting phorbol-12,13-diesters in canine kidney (MDCK) cells. J. Biol. Chem. 253:4783-4790.

21. Levine, L., and H. Fujiki. 1985. Stimulation of arachidonic acid metabolism by different types of tumor promoters. Carcinogenesis. 6: 1631-1634.

22. Grantham, J. J., and J. Orloff. 1968. Effect of prostaglandin $E_{1}$ on the permeability response of the isolated collecting tubule to vasopressin, adenosine 3',5'-monophosphate, and theophylline. J. Clin. Invest. 47:1154-1161.

23. Nadler, S. P., S. C. Hebert, and B. M. Brenner. 1985. PGE , $^{2}$ forskolin, and cholera toxin interactions in rabbit cortical collecting tubule. Am. J. Physiol. 250:F127-F135.

24. Schlondorff, D., C. P. Carvounis, M. Jacoby, J. A. Satriano, and S. D. Levine. 1981. Multiple sites for interaction of prostaglandin and vasopressin in toad bladder. Am. J. Physiol. 241:F625-F631.

25. De Reimer, S. A., J. A. Strong, K. A. Albert, P. Greengard, and L. K. Kaczmarek. 1984. Enhancement of calcium current in Aplysia neurons by phorbol ester and protein kinase C. Nature (Lond.). 313: 313-315.

26. Kurtz, A., J. Pfeilschifter, A. Hutter, C. Buerle, R. Nobiling, R. Traugner, E. Hackenthal, and C. Bauer. 1986. Role of protein kinase C in inhibition of renin release caused by vasoconstrictors. Am. J. Physiol. 250:C563-C571.

27. Hardy, M. A. 1978. Intracellular calcium as a modulator of transepithelial permeability to water in frog urinary bladder. J. Cell Biol. 76:787-791.

28. Moolenaar, W. H. 1986. Effects of growth factors on intracellular pH regulation. Ann. Rev. Physiol. 48:363-376.

29. Busa, W. B., and R. Nuccitelli. 1984. Metabolic regulation via intracellular pH. Am. J. Physiol. 246:R409-438.

30. Brem, A. S., E. Eich, M. Pearl, and A. Taylor. 1985. Anion transport inhibitors: effect on water and sodium transport in the toad urinary bladder. Am. J. Physiol. 248:F594-F601.

31. L'Allemain, G., A. Franchi, E. Cragoe, Jr., and J. Pouyssegur. 1984. Blockade of the $\mathrm{Na}^{+} / \mathrm{H}^{+}$antiport abolishes growth factor-induced DNA synthesis in fibroblasts: structure-activity relationship in the amiloride series. J. Biol. Chem. 259:4313-4319. 